\title{
Torque Ripple Investigation in Squirrel Cage Induction Machines
}

\author{
Alessandro Marfoli, Giacomo Sala, Luca Papini, Paolo Bolognesi, Chris Gerada \\ Power Electronics, Machines and Control Group \\ University of Nottingham \\ Nottingham, UK
}

\begin{abstract}
Low torque ripple of electrical machines is an important requirement for many different applications. The causes of torque fluctuations in squirrel cage asynchronous induction motors might be a challenging task due to the complex phenomena raised by the whole set of rotor bars. This paper proposes an accurate analysis of the torque contributions distinguishing in time-varying, synchronous and asynchronous components. The investigation is performed by adopting a general analytical approach based on a circuital modelling technique for electromagnetic devices. The analysis aims to identify spatial harmonic interactions between stator and rotor magneto-motive forces which generate torque components. The most interesting effects obtained from the analytical investigation are compared and validated against finite element results for the most significant operating points.
\end{abstract}

Index Terms-Analytical models, Harmonic analysis, Induction motors, Torque Ripple

\section{INTRODUCTION}

Various methodologies for the early stage design of Squirrel Cage Induction Motors (SCIMs) are reported in literature and widely adopted. The success of these approaches is related to the use of simplified mathematical models to describe the complex phenomena which permit to describe the electro-magnetomechanical energy transformation. However, such approaches are often based on the fundamental component of the main quantities such as magneto-motive forces $(\mathscr{M})$, flux density $\left(B_{\text {gap }}\right)$ and currents $(\bar{i})$. Whereas the fundamental harmonic enables an accurate prediction of the performance in term of rated torque at a given operating point, there is a lack of informations about high order harmonic interactions between stator and rotor and, consequently, on the currents and torque oscillations. To fully understand the SCIM behaviours a circuital representation able to account such high order harmonics raised by stator windings and rotor cage, is required. A general approach to model electromagnetic devices is presented in [1] and [2] where, under simplified assumptions, the integral form of the Maxwell equations are applied to the region where most of the electromechanical conversion take place, namely the main rotor-stator air gap clearance. Application examples of such approach are presented in [3] and [4] addressing synchronous generators and in [5] referring to SCIMs. In [6] an example of modelling the rotor cage based on the concept of the Winding Functions (WFs) is presented and a derivation of a new $d-q$ axes model is introduced. A detailed analytical steady state analysis of the harmonic field effects in SCIMs is presented in [7], where plural phenomenon are considered. Although, a detailed experimental investigation to identify asynchronous and synchronous torques is reported in [7], addressing three stator combinations each one featured by 19 rotor topologies, the causes of torque components and the logical correlation between ripple and synchronous torques are not completely investigated and highlighted.

In this paper an analytical revision of the torque components acting in SCIMs is proposed based on a general circuital model representation described in [5]. The main goal of this study is to provide in depth the analysis of the torque ripple phenomena yet using a simplified approach. It has been possible to confirm some of the conclusions presented in [7] and extend the analysis to determine the spatial harmonic interactions which mainly contribute to ripple, synchronous and asynchronous torque components. The adopted approach is presented in a generic form that can be applied to any number of rotor bars. The analytical conclusion are compared against Finite Element (FE) results.

\section{BACKGROUND}

This section introduces the general analytical equations which define the electro-magneto-mechanical behaviour of electrical machines. According with the main hypotheses of magnetically linear materials and negligible drop of magnetomotive forces in the magnetic cores, the system of equations which governs the electromagnetic and mechanical phenomena can be expressed as (1) and (2), respectively.

$$
\begin{gathered}
\bar{v}(t)=\mathbf{R} \bar{i}(t)+\frac{d \alpha(t)}{d t} \frac{\partial \mathbf{L}(\alpha)}{\partial \alpha} \bar{i}+\mathbf{L}(\alpha) \frac{d \bar{i}(t)}{d t} \\
W(\alpha, \bar{i})=\frac{\partial C(\alpha, \bar{i})}{\partial \alpha}=\frac{1}{2} \bar{i}^{T} \frac{\partial \mathbf{L}(\alpha)}{\partial \alpha} \bar{i}
\end{gathered}
$$

In (1) and (2) $\alpha$ is the position variable representing the angular displacement between stator and rotor, $\bar{i}$ is the vector of the currents flowing in the machine phases, $\mathbf{R}$ is the resistances matrix, $\mathbf{L}(\alpha)$ is the inductances matrix, $\bar{v}$ is the vector of the voltages imposed to the machine phases, $C$ is the electromagnetic coenergy and $W$ is the electromagnetic torque tending to increase the angular displacement $\alpha$ (motor operation). The SCIM here considered is equipped with a symmetrical three-phase stator winding and a symmetrical rotor squirrel-cage featuring $b$ bars per stator pole pair. The rotor bars are modelled as a set of $b$ equivalent phases, each 
one representing a loop delimited by adjacent bars according to [5]. Eq (1) can be then re-written highlighting the stator and rotor components, leading to (6), where the voltages of the cage loops are $\bar{v}_{r}=0$. The equations system related to the rotor cage can be separated as in (7). Considering an isotropic machine structure, i.e. neglecting the effects of the slot openings, the matrix of rotor inductances $\mathbf{L}_{\mathbf{r r}}$ turns out composed by constant parameters and the expression (7) can be simplified as in (3) where the term $\frac{d \bar{\varphi}\left(\alpha, \bar{t}_{s}\right)}{d t}$ can be represented as in (4).

$$
\begin{gathered}
\overline{0}=\mathbf{R}_{\mathbf{r}} \overline{i_{r}}+\mathbf{L}_{\mathbf{r r}} \frac{d \overline{i_{r}}}{d t}+\frac{d \bar{\varphi}\left(\alpha, \bar{i}_{s}\right)}{d t} \\
\frac{d \bar{\varphi}\left(\alpha, \bar{i}_{s}\right)}{d t}=\mathbf{L}_{\mathbf{r s}}(\alpha) \frac{d \bar{i}_{s}}{d t}+\frac{d \alpha}{d t} \frac{\partial \mathbf{L}_{\mathbf{r s}}(\alpha)}{\partial \alpha} \bar{i}_{s}
\end{gathered}
$$

\section{STATOR AND ROTOR MAGNETO-MOTIVE FORCES}

The balanced three phase currents circulating in a symmetric set of three stator phases can be represented as in (5).

$$
i_{s, \xi}(t)=I_{s} \sin \left(w_{s} t-\frac{2 \pi \xi}{3}\right) \quad \text { with } \quad \xi=1,2,3
$$

Considering an isotropic structure, the stator magneto-motive force $\mathscr{M}_{s}$ can be written as a product between the harmonics summation of the stator winding functions representative of the coils distribution and the corresponding currents giving in turns after manipulation the form reported in (8), where $\gamma$ is the coordinate in the stator angular reference frame, $\bar{N}_{s}$ is the vector composed by the stator WF phases, $p$ is the number of pole pairs, $w_{s}$ is the angular pulsation of the stator currents and ${ }^{6 h \pm 1} \gamma_{s}$ is the displacement of each spatial harmonic. The stator magneto-motive force $\mathscr{M}_{s}$ can be represented as a summation of forward travelling spatial harmonics of $6 h+1$ order and backward travelling spatial harmonics of $6 h-1$ order. Assuming that the rotor rotates at constant speed according to $\alpha(t)=w_{s} t(1-s)$, where $s$ is the slip, the flux linked to the $q^{\text {th }}$ rotor coil loop, raised by the stator currents, can be calculated by applying (10), where
$B_{g s}$ is the flux density in the air-gap imposed by the stator currents, $r_{g}$ is the air-gap radius, $l$ is the stack length of the machine, $\epsilon_{g}$ is the air-gap thickness and $N_{r, q}$ is the winding function related to the $q^{t h}$ loop.

$$
\begin{array}{r}
\psi_{q}(t)=l r_{g} \int_{0}^{2 \pi} B_{g s}\left(\bar{i}_{s}(t), \gamma\right) N_{r, q}(\gamma, \alpha(t)) d \gamma \\
B_{g s}\left(\bar{i}_{s}, \gamma\right)=\mu_{0} \frac{\mathscr{M}_{s}\left(\overline{i_{s}}, \gamma\right)}{\epsilon_{g}}=\mu_{0} \frac{\bar{N}_{s}(\gamma)^{T} \overline{i_{s}}}{\epsilon_{g}}
\end{array}
$$

It is important to note that in case of isotropic structure the spatial spectrum of $B_{g s}$ features only the harmonics composing the spectrum of $\mathscr{M}_{s}$. The WF $N_{r, q}$ of a generic $q$ rotor loop reported in (10) can be represented as a spatial harmonics decomposition according to (12).

$$
N_{r, q}(\gamma, \alpha)=\sum_{k=0}^{\infty}{ }^{k} N_{r} \cos \left(k p \gamma-k(1-s) w_{s} t-\frac{2 \pi k}{b} q-{ }^{k} \gamma_{r}\right)
$$

When substituting (12) in (10), due to the effect of integration from 0 to $2 p i$ only the matching spatial harmonics in (8) and (12) produce a contribution to the flux linkage, thus providing an excitation term to (3). Since the latter is a linear-differential equation with constant parameters with respect to $\bar{i}_{r}$, at steady state the rotor current waveforms feature a periodic trend whose spectrum includes only the time harmonics provided by (4), i.e. the ones matching the above condition. Therefore, keeping into account the assumed symmetry of the rotor cage, the currents flowing in the cage loops at steady state are expected to consist in a symmetrical set as in (9), where the $q^{t h}$ loop current is highlighted and ${ }^{6 h \pm 1} I_{r}$ and ${ }^{6 h \pm 1} \gamma_{s r}$ assume the appropriate values. The magneto-motive force $\mathscr{M}_{r}=\bar{N}_{r}(\gamma)^{T} \bar{i}_{r}$ correlated with this group of currents can be calculated as in (15), where $\tilde{h}=(6 h \pm 1)=1,5,7,11,13, \ldots$. A simplified exponential form can be written rearranging (15) as in (16) where it can be observed that the two sums over $q$ produce a not zero result when $k \mp \tilde{h}=z b$ with $z \in Z$, respectively. Furthermore, both the summations can be not zero for the same $k, \tilde{h}$ values when $b$ is even and both $k$

$$
\begin{gathered}
{\left[\begin{array}{c}
\overline{v_{s}} \\
\overline{0}
\end{array}\right]=\left[\begin{array}{c}
\mathbf{R}_{\mathbf{s}} \\
0
\end{array}\right] \bar{i}_{s}+\left[\begin{array}{c}
0 \\
\mathbf{R}_{\mathbf{r}}
\end{array}\right] \bar{i}_{r}+\left[\begin{array}{c}
\mathbf{L}_{\mathbf{s s}}(\alpha) \\
\mathbf{L}_{\mathbf{r s}}(\alpha)
\end{array}\right] \frac{d \bar{i}_{s}}{d t}+\frac{d \alpha}{d t}\left[\frac{\partial \mathbf{L}_{\mathbf{s}}(\alpha)}{\partial \alpha} \frac{\partial \mathbf{L}_{\mathbf{r s}}(\alpha)}{\partial \alpha}\right] \bar{i}_{s}+\left[\begin{array}{c}
\mathbf{L}_{\mathbf{s r}}(\alpha) \\
\mathbf{L}_{\mathbf{r r}}(\alpha)
\end{array}\right] \frac{d \bar{i}_{r}}{d t}+\frac{d \alpha}{d t}\left[\frac{\partial \mathbf{L}_{\mathbf{s r}}(\alpha)}{\partial \alpha} \frac{\partial \mathbf{L}_{\mathbf{r r}}(\alpha)}{\partial \alpha}\right] \bar{i}_{r}} \\
\overline{0}=\mathbf{R}_{\mathbf{r}} \overline{\overline{i_{r}}}+\mathbf{L}_{\mathbf{r r}}(\alpha) \frac{d \overline{i_{r}}}{d t}+\frac{d \alpha}{d t} \frac{\partial \mathbf{L}_{\mathbf{r r}}(\alpha)}{\partial \alpha} \bar{i}_{r}+\mathbf{L}_{\mathbf{r s}}(\alpha) \frac{d \bar{i}_{s}}{d t}+\frac{d \alpha}{d t} \frac{\partial \mathbf{L}_{\mathbf{r s}}(\alpha)}{\partial \alpha} \bar{i}_{s} \\
\mathscr{M}_{s}\left(\bar{i}_{s}(t), \gamma\right)=\bar{N}_{s}(\gamma)^{T} \overline{i_{s}}(t)=\sum_{h=0}^{\infty}(6 h \pm 1) \mathscr{M}_{s}\left(I_{s}\right) \cos \left((6 h \pm 1) p \gamma \mp w_{s} t-{ }^{6 h \pm 1} \gamma_{s}\right) \\
i_{r, q}(t)=\sum_{h=0}^{\infty}{ }^{6 h \pm 1} I_{r} \sin \left([\mp 1+(6 h \pm 1)(1-s)] w_{s} t+\frac{2 \pi q}{b}(6 h \pm 1)+{ }^{6 h \pm 1} \gamma_{s r}\right) \text { with } q=1, \ldots, p b
\end{gathered}
$$


and $\tilde{h}$ are odd multiples of $b / 2$. In other words, for the spatial harmonic of order multiple of $b / 2$, the rotor reaction is described by a pulsating magneto-motive force contribution.

\section{TORQUE RIPPLE ANALYSIS}

In this section, the interactions between stator and rotor spatial harmonics are investigated, aiming to assess their impact on the torque components. The torque of a generic electrical machine can be calculated as the derivative of the coenergy $C(\alpha, \bar{i})$ with respect to the position $\alpha$ at fixed currents $\bar{i}$, as reported in [1]. In a magnetically-linear scenario, $C(\alpha, \bar{i})$ can be calculated either from the inductance matrix as in (13) or integrating the electromagnetic energy density stored in the air gap volume as in (14).

$$
\begin{gathered}
W(\alpha, \bar{i})=\frac{\partial C(\alpha, \bar{i})}{\partial \alpha}=\frac{1}{2} \bar{i}^{T} \frac{\partial L(\alpha)}{\partial \alpha} \bar{i} \\
W(\alpha, \bar{i})=\frac{\partial}{\partial \alpha}\left\{\frac{1}{2} \iiint B_{\text {gap }} H_{\text {gap }} d V\right\}
\end{gathered}
$$

For analytical purposes, the second expression of the torque, (14), is taken into account because it does not require the computation of the inductance matrix and it allows to better link the harmonic interactions between the stator and rotor magneto-motive forces, namely $\mathscr{M}_{s}$ and $\mathscr{M}_{r}$. Eq. (14) can be rearranged as (17), taking the derivative with respect to the rotor angular position $\alpha$ inside the integral. Substituting
$\mathscr{M}_{s}$ and $\mathscr{M}_{r}$ with the corresponding expressions (8) and (16), the torque contributions can be represented in a compact form as reported in (18). The integral result of the first and second components, considering the stator $\xi^{t h}$ and the rotor $q^{\text {th }}$ terms, are reported in (19) and (20), respectively. Also in this case, following the procedure used fo the analysis of the rotor magneto-motive contributions, only the spatial harmonic components having the same order can possibly lead to a not zero result. The second term of the torque expression, (18), can be written in the symbolic form: $x^{T} A x$, where the matrix $A$ is composed by the elements coming from (20). Since such matrix is actually anti-symmetric $\left(A^{T}=-A\right)$, the quadratic product $x^{T} A x=0$. This result confirms that in a isotropic scenario the currents circulating only in the rotor bars cannot produce any torque component: therefore, only the first term of (18) has an impact on the torque. The last step to achieve the final expression of the torque is multiplying the matrix, resulting from (19) by the stator and rotor current vectors, where the latter is expressed as in (9). After further manipulation, the torque expression turns out to be composed by four different terms as shown in (23). Each one featured by a different coefficient defining the speed pulsation of the relative component according to (21) and (22).

$$
\begin{array}{ll}
\mathscr{A}_{1}=(v-k)(1-s) \mp 1-1 & \mathscr{A}_{2}=(v-k)(1-s) \mp 1+1 \\
\mathscr{A}_{3}=(v+k)(1-s) \mp 1+1 & \mathscr{A}_{4}=(v+k)(1-s) \mp 1-1
\end{array}
$$

$$
\begin{aligned}
& \mathscr{M}_{r}(\bar{i}, \gamma)=\sum_{q=1}^{p b} \sum_{k=0}^{\infty} \sum_{\tilde{h}}{ }^{k} N_{r}{ }^{\tilde{h}} I_{r} \cos \left(k p \gamma-k(1-s) w_{s} t-\frac{2 \pi k}{b} q-{ }^{k} \gamma_{r}\right) \sin \left([\mp 1+\tilde{h}(1-s)] w_{s} t+\frac{2 \pi \tilde{h}}{b} q+{ }^{\tilde{h}} \gamma_{r s}\right) \\
& \mathscr{M}_{r}(\bar{i}, \gamma)=\sum_{k=0}^{\infty} \sum_{\tilde{h}} \frac{{ }^{k} N_{r} \tilde{h}}{2} I_{r} \operatorname{Im}\left\{e^{j\left(k p \gamma+[(\tilde{h}-k)(1-s) \mp 1] w_{s} t-{ }^{k} \gamma_{r}+{ }^{\tilde{h}} \gamma_{r s}\right)} \sum_{q=1}^{p b}\left[e^{-j 2 \pi \frac{k-\tilde{h}}{b}}\right]^{q}-\right.
\end{aligned}
$$

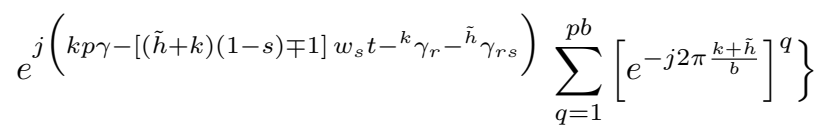

$$
\begin{aligned}
& W(\alpha, \bar{i})=\frac{\partial}{\partial \alpha}\left\{\frac{l \epsilon_{g} r_{g}}{2 \mu_{0}} \int_{0}^{2 \pi} B_{g a p}^{2}(\alpha, \gamma) d \gamma\right\}=\frac{l \mu_{0} r_{g}}{\epsilon_{g}} \int_{0}^{2 \pi}\left(\mathscr{M}_{s}\left(\gamma, \bar{i}_{s}\right)+\mathscr{M}_{r}\left(\gamma, \alpha, \bar{i}_{r}\right)\right) \frac{\partial \mathscr{M}_{r}\left(\gamma, \alpha, \bar{i}_{r}\right)}{\partial \alpha} d \gamma \\
& W(\alpha, \bar{i})=\frac{l \mu_{0} r_{g}}{\epsilon_{g}} \int_{0}^{2 \pi}\left[{\overline{i_{s}}}^{T} \bar{N}_{s}(\gamma) \frac{\partial \bar{N}_{r}(\gamma, \alpha)^{T}}{\partial \alpha}{\overline{i_{r}}}+{\overline{i_{r}}}^{T} \bar{N}_{r}(\gamma, \alpha) \frac{\partial \bar{N}_{r}(\gamma, \alpha)^{T}}{\partial \alpha} \overline{i_{r}}\right] d \gamma \\
& \int_{0}^{2 \pi} N_{s, \xi}(\gamma) \frac{\partial N_{r, q}(\gamma, \alpha)}{\partial \alpha}=\pi \sum_{k=1}^{\infty} k \cdot{ }^{k} N_{s, \xi}{ }^{k} N_{r, q} \sin \left(k w_{r} t-{ }^{k} \gamma_{r s}\right) \\
& \int_{0}^{2 \pi} N_{r, q}(\gamma, \alpha) \frac{\partial N_{r, \eta}(\gamma, \alpha)}{\partial \alpha}=\pi \sum_{h=1}^{\infty} h \cdot{ }^{h} N_{r, q}{ }^{h} N_{r, \eta} \sin \left(\frac{2 \pi h}{b}(\eta-q)\right)
\end{aligned}
$$




$$
\begin{aligned}
W(\alpha, \bar{i}) & =\frac{\pi l \mu_{0} r_{g}}{4 \epsilon_{g}} \sum_{k=1}^{\infty} \sum_{v=0}^{\infty} k^{6 v \pm 1} I_{r} I_{s}{ }^{k} N_{s}{ }^{k} N_{r} \operatorname{Re}\{ \\
& {\left[e^{j\left(w_{s} \mathscr{A}_{1} t-^{v} \gamma_{N_{s r}}\right)} \sum_{i=0}^{2} e^{-j \frac{2 \pi}{3} i(1+k)} \sum_{q=1}^{p b} e^{j \frac{2 \pi q}{b}(v-k)}\right]+\left[e^{j\left(w_{s} \mathscr{A}_{2} t+^{v} \gamma_{N_{s r}}\right)} \sum_{i=0}^{2} e^{-j \frac{2 \pi}{3} i(1-k)} \sum_{q=1}^{p b} e^{-j \frac{2 \pi q}{b}(v-k)}\right]-} \\
& {\left.\left[e^{j\left(w_{s} \mathscr{A}_{3} t+^{v} \gamma_{N_{s r}}\right)} \sum_{i=0}^{2} e^{-j \frac{2 \pi}{3} i(1+k)} \sum_{q=1}^{p b} e^{j \frac{2 \pi q}{b}(v+k)}\right]-\left[e^{j\left(w_{s} \mathscr{A}_{4} t+{ }^{v} \gamma_{N_{s r}}\right)} \sum_{i=0}^{2} e^{-j \frac{2 \pi}{3} i(1-k)} \sum_{q=1}^{p b} e^{-j \frac{2 \pi q}{b}(v+k)}\right]\right\} }
\end{aligned}
$$

\section{CASE STUdy}

The analytical investigation introduced so far is general and can be potentially adopted for any number of bars, stator winding layout and easily extended also for multi-three phase configurations. As proof of the concepts, the conclusive equations are applied to a 3-phase SCIM with a rated power of $11 \mathrm{~kW}, 2$ pole pairs, distributed single layer stator winding ( $\mathrm{Y}$ connected) with 3 stator slots per pole per phase and equipped with a set of 14 rotor bars per pole pair $(b=14)$.

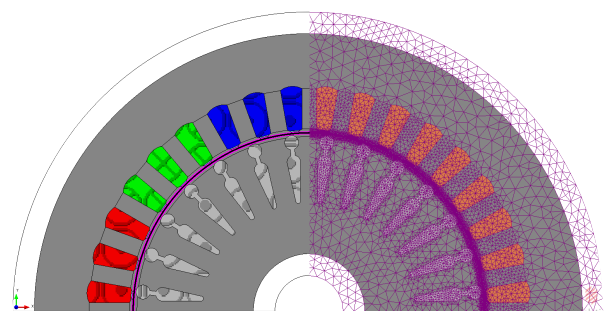

Figure 1. Motor under investigation: structure and mesh layout

\section{RESUlTS}

For the considered case study, the equations resulting from the general analytical model have been implemented in order to evaluate the behaviour of the all torque contributions. Even though, the amplitude and frequency values of the torque harmonic amplitudes are affected by the operating point (defined by the slip value, $s$ ), the stator and rotor spatial harmonic interactions responsible of such components do not change as they depend only by the geometrical motor layout: windings arrangement and number of bars. Figure 2 and Figure 4 show a graphical illustration of the harmonic contributions included in (8), (9), (16) and (23) at $s=0.02$ (rated slip) and $s=6 / 7$, when the stator windings are supplied at rated frequency $(f=50 \mathrm{~Hz})$. All the quantities represented in the figures, except for the first column, are normalized with respect to the fundamental pulsation of the rotor current: $w_{r}=2 \pi f_{r}$ with $f_{r}=1 \mathrm{~Hz}$. In the first column to the left, the spatial harmonic orders of $\mathscr{M}_{s}$ and their travelling speeds are reported: the red points represent the forward rotating harmonics and the blu points the backward ones. In the second column the green
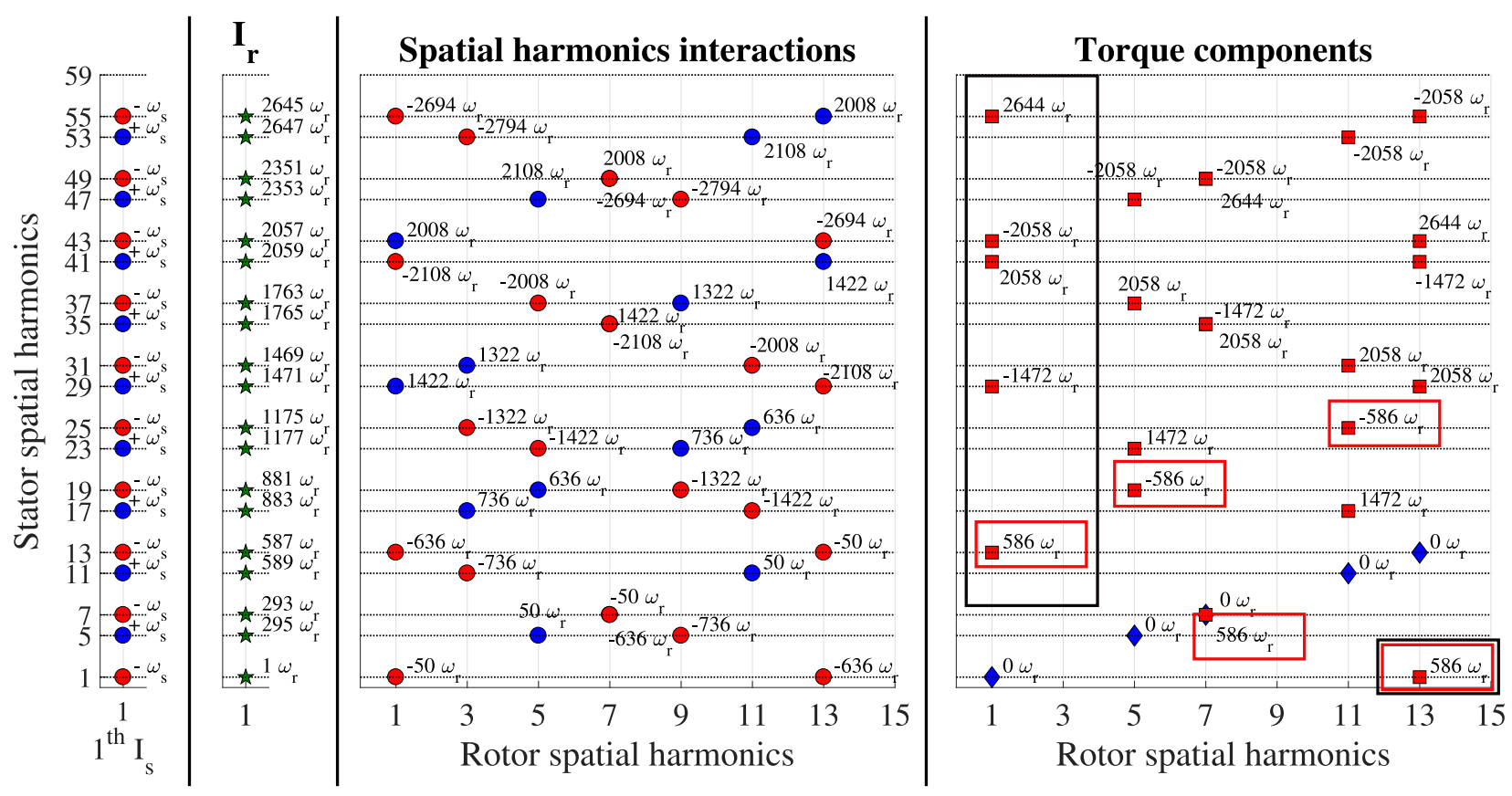

Figure 2. Harmonic orders investigation: Stator magneto-motive force $\mathscr{M}_{s}$, rotor current $i_{j}$, rotor magneto-motive force $\mathscr{M}_{r}$ and torque $W(\alpha, \bar{i}):$ Slip $=0.02$ 


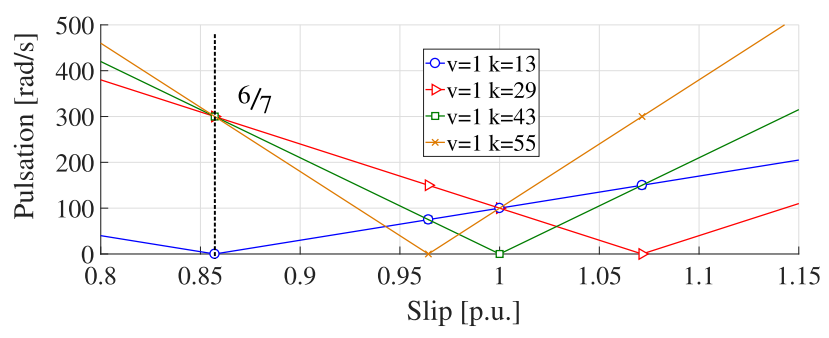

Figure 3. Ripple pulsation due to different spatial harmonic orders interaction.

stars markers indicate the pulsations of the currents induced in the rotor loops, correlated to each stator spatial harmonic. The third column shows the travelling speeds of each spatial harmonic of the rotor magneto-motive force, $\mathscr{M}_{r}$ produced by the related stator spatial harmonics: the red points highlight the forward travelling waves, whereas the blue represents the backward ones. In the last column, the markers are located where stator and rotor spatial harmonics of the magnetomotive forces interact with each other leading to torque components: the blue diamond markers represent harmonic interactions producing a non-zero average torque, whereas the red square markers represent ripple components. It is worth to observe that each $(6 h \pm 1)^{t h}$ stator spatial harmonic induces the rotor to react with a spatial harmonic of the same order, rotating at the same speed and direction, leading to a non-zero average torque. In addition, due to the number of rotor bars under each pole pair $(b=14)$, each $7^{t h}, 35^{t h}, 49^{t h}, \ldots$ harmonic gives rise two component of rotor magneto-motive forces and therefore two torque components. Finally, independently from the operating condition, each harmonic of the stator spatial magneto-motive force generates a torque ripple contribution interacting with all the rotor magneto-motive forces having the same space spatial periodicity but raised by stator spatial harmonics with a different order. These last interactions exist because the rotor bars are not infinite, and the cage reaction introduces new frequency components in the space harmonics of the magnetic field in the airgap. For example, in Figure 2, the $13^{\text {th }}$ order harmonic of $\mathscr{M}_{s}$ (first column) induces in the rotor a current at $587 w_{r}$ (second column) which creates a $1^{\text {st }}$ order harmonic of $\mathscr{M}_{r}$ rotating at $-636 w_{r}$ speed (third column). This spatial harmonic interacts with a same order stator spatial harmonic which rotates at $-50 w_{r}$, producing a torque pulsation at $w_{\text {ripple }}=-636 w_{r}+50 w_{r}=-586 w_{r}$ (fourth column). The same concept can be repeated for the all the harmonics of the stator magneto-motive force.

Different combinations of harmonic orders can be considered as part of the same group, which contributes to the same ripple frequencies, as highlighted in red squares for $w_{\text {ripple }}=$ $-586 w_{r}$, in Figure 2. However, it is reasonable expecting that the most significant rotor or stator spatial harmonics producing torque ripple are the ones interacting with the rotor or stator fundamental magneto-motive force, circled in black, in Figure 2.

Equation (23) permits also to analyse the ripple pulsations according with the slip value. In Figure 3 such behaviour is shown by highlighting the harmonic interactions between the main stator spatial harmonics and the first rotor spatial harmonic. From this analysis it is possible to describe an additional phenomenon. In particular, given a group of harmonic interactions producing a ripple torque component at nominal speed, the same interactions generate an average torque component for a specific value of the rotor speed. These
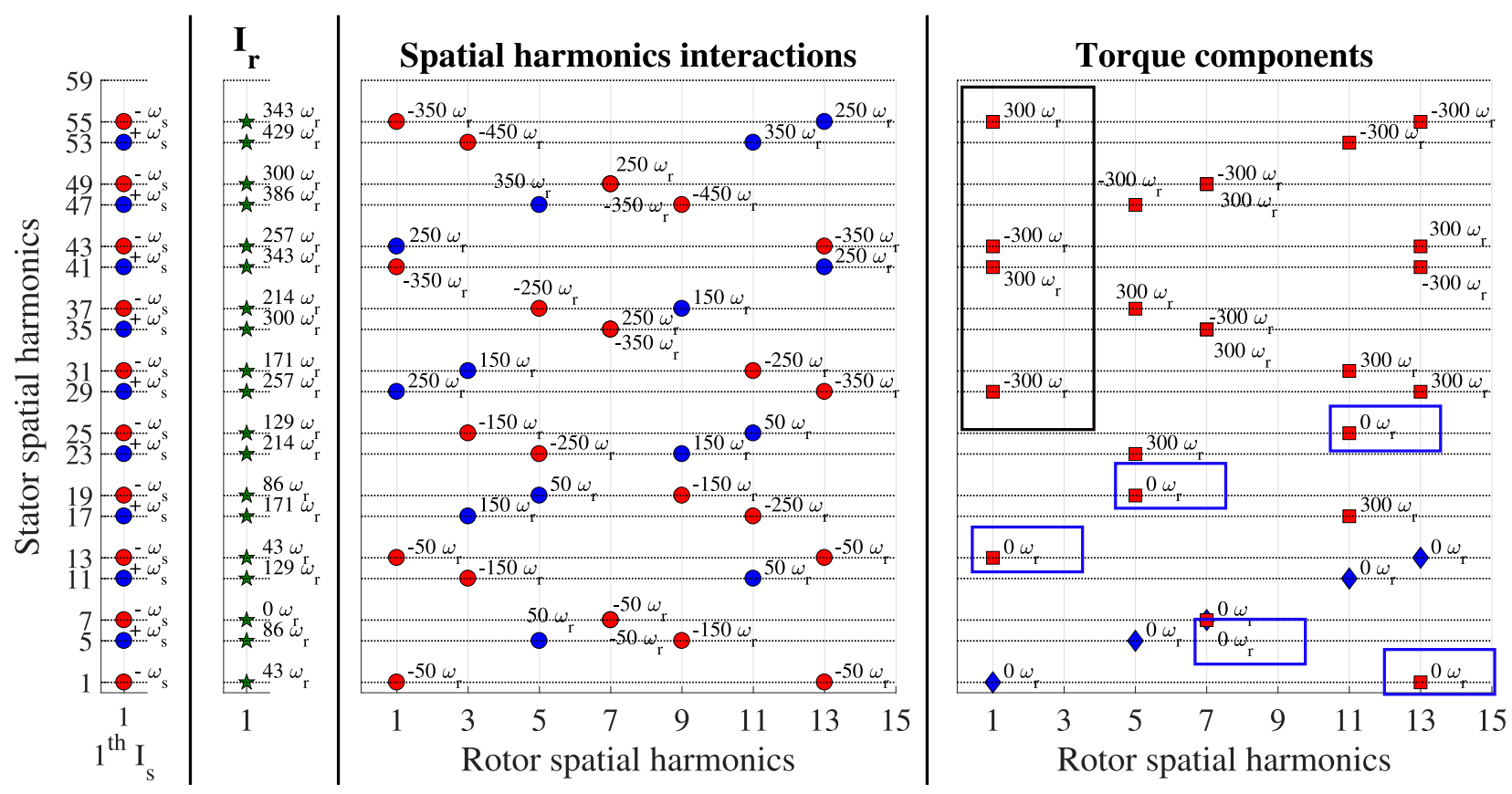

Figure 4. Harmonic orders investigation: Stator magneto-motive force $\mathscr{M}_{s}$, rotor current $i_{j}$, rotor magneto-motive force $\mathscr{M}_{r}$ and torque $W(\alpha, \bar{i}):$ Slip $=6 / 7$ 
torque contributions are also known with the name of "synchronous torques" [7]. In fact, from a detailed analysis of the equations given by the model, it is possible to note that these interactions generate a torque ripple that gradually reduces its frequency until the rotor speed reaches the synchronising value for the considered family of torque interactions. For example, as shown in Figure 3, the first rotor harmonic produced by the $13^{\text {th }}$ stator harmonic, at slip $=6 / 7$, is synchronized with the first stator harmonic giving in turns an average torque value while for $\operatorname{slip} \neq 6 / 7$ the interaction result in a torque ripple oscillation. This phenomena is in common for all the harmonics part of the group producing at rated slip the torque oscillation at $w_{\text {ripple }}=-586 w_{r}$ (circled in red, in Figure 2) and an average torque at slip $=6 / 7$ (circled in blue, in Figure 4). The same behaviour is repeated for all the harmonic groups raising the same torque pulsation at rated slip which end up synchronized at different slip values.

The analytical conclusions reported above, have been validated by FE transient analysis. In Figure 5, results provided by the simulations are displayed for the rated operating condition $s=0.02$ and for $s=6 / 7$ in term of rotor bar current waveforms and torque profile with their respective spectra. It can be noticed that the frequencies of both bar current and torque ripple were correctly predicted by the theoretical analysis, for both the operating condition, confirming the validity of the analytical model.

\section{CONCLUSION}

In this paper, an in-deep analysis of the torque contributions in squirrel cage induction machines has been carried out. The study was based on a revision of the analytical expressions used to describe the harmonic interactions between stator and rotor. The equations of the magneto-motive force of both stator and rotor windings have been analysed, describing the torque components and highlighting the harmonic orders interactions that generate them. The causes of the torque ripple components are identified and their correlations with the synchronous
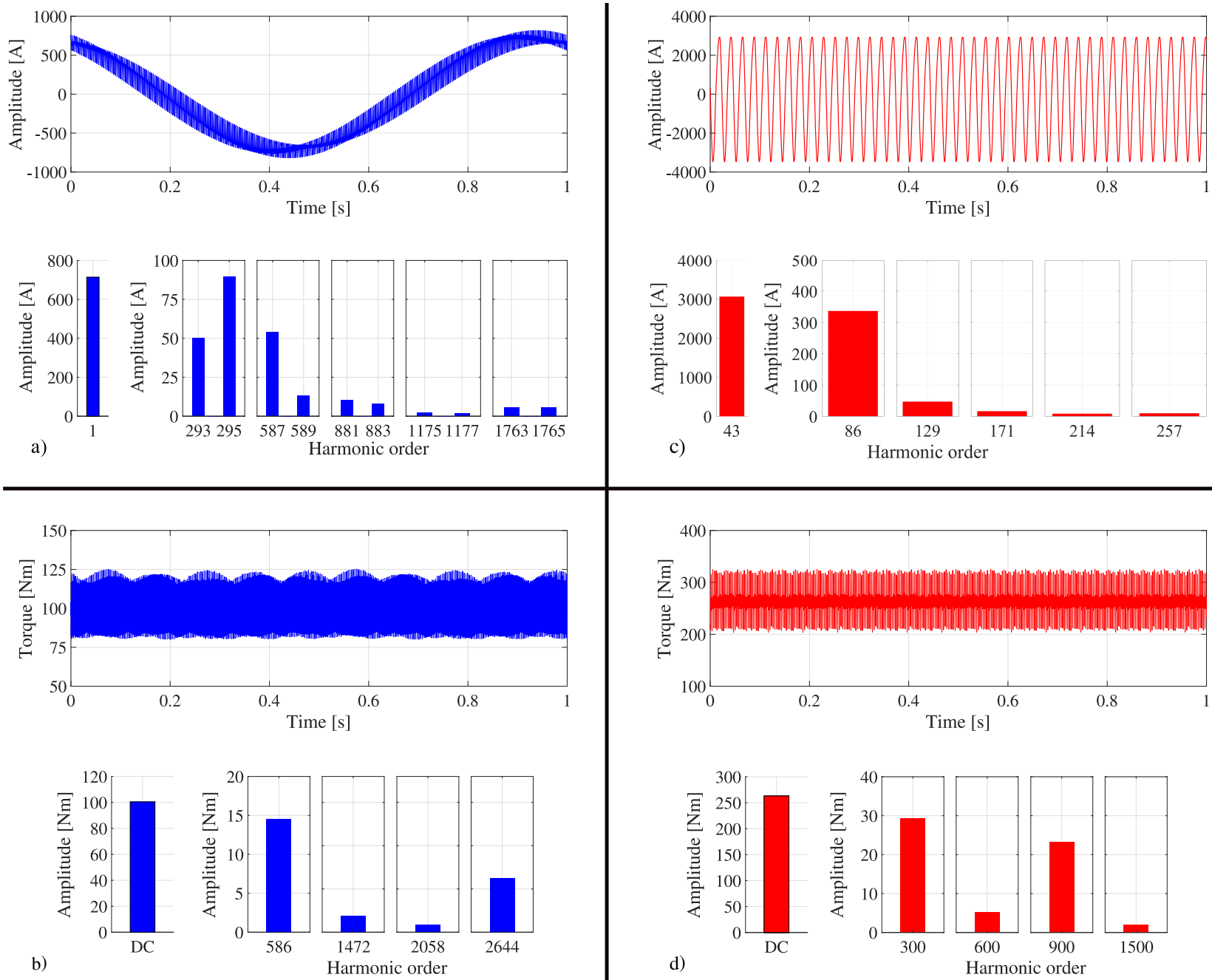

Figure 5. Linear FE results: a) Rotor bar current waveform and spectrum at slip $=0.02$ b) torque profile and spectrum at slip $=0.02$ c) Rotor bar current waveform and spectrum at slip $=6 / 7 \mathrm{~b}$ ) torque profile and spectrum at slip $=6 / 7$ 
torques are pointed out. The analytical model was applied to a particular case study of induction machine, and validated by means of FE simulations. The comparison of the results for different operating points exhibits a perfect agreement between the analytical and the FE model, in terms of both rotor bar current and torque spectra.

\section{ACKNOWLEDGMENT}

The financial support from ABB Corporate Research in Västerảs for this research is gratefully acknowledged.

\section{REFERENCES}

[1] P. Bolognesi, "Generalized circuital modeling of electromagnetic devices," Proc. of ICEM 2004 Conf., Cracow, Sep. 2004., 2004.

[2] P. Bolognesi, "A mid-complexity analysis of long-drum-type electric machines suitable for circuital modeling," in 2008 18th International Conference on Electrical Machines, Sept 2008, pp. 1-5.

[3] S. Nuzzo, P. Bolognesi, M. Galea, and C. Gerada, "A hybrid analyticalnumerical approach for the analysis of salient-pole synchronous generators with a symmetrical damper cage," in 2017 IEEE International Electric Machines and Drives Conference (IEMDC), May 2017, pp. 1-8.

[4] S. Nuzzo, P. Bolognesi, C. Gerada, and M. Galea, "Simplified damper cage circuital model and fast analytical-numerical approach for the analysis of synchronous generators," IEEE Transactions on Industrial Electronics, pp. 1-1, 2018.

[5] A. Marfoli, P. Bolognesi, L. Papini, and C. Gerada, "Mid-complexity circuital model of induction motor with rotor cage: A numerical resolution," in 2018 XIII International Conference on Electrical Machines (ICEM), Sept 2018, pp. 277-283.

[6] A. R. Munoz and T. A. Lipo, "Complex vector model of the squirrelcage induction machine including instantaneous rotor bar currents," IEEE Transactions on Industry Applications, vol. 35, no. 6, pp. 1332-1340, Nov 1999.

[7] B. Heller and V.Hamata, Harmonic field effects in Induction Machine, N. Y. Elsevier, Ed., 1977. 